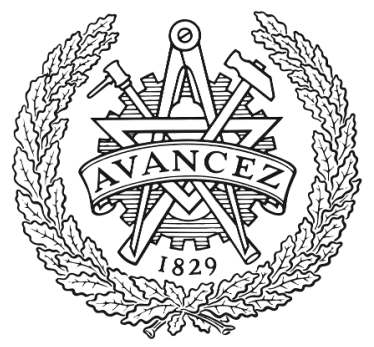

CHALMERS

UNIVERSITY OF TECHNOLOGY

\title{
Double doping of conjugated polymers with monomer molecular dopants
}

Downloaded from: https://research.chalmers.se, 2023-04-26 14:43 UTC

Citation for the original published paper (version of record):

Kiefer, D., Kroon, R., Hofmann, A. et al (2019). Double doping of conjugated polymers with monomer molecular dopants. Nature Materials, 18(2): 149-155.

http://dx.doi.org/10.1038/s41563-018-0263-6

N.B. When citing this work, cite the original published paper. 


\title{
Double doping of conjugated polymers with monomer molecular dopants
}

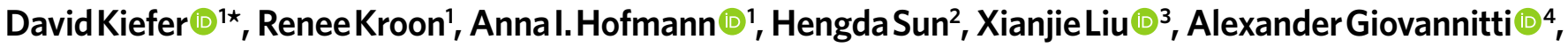 \\ Dominik Stegerer 1,5, Alexander Cano', Jonna Hynynen', Liyang Yu1, Yadong Zhang'6 , Dingqi Nai', \\ Thomas F. Harrelson ${ }^{7}{ }^{7}$, Michael Sommer ${ }^{5}$, Adam J. Moulé7, Martijn Kemerink ${ }^{8}{ }^{8}$, Seth R. Marder ${ }^{6}$, \\ lain McCulloch ${ }^{4,9}$, Mats Fahlman ${ }^{3}$, Simone Fabiano (102 and Christian Müller (1) ${ }^{1 \star}$
}

\begin{abstract}
Molecular doping is a crucial tool for controlling the charge-carrier concentration in organic semiconductors. Each dopant molecule is commonly thought to give rise to only one polaron, leading to a maximum of one donor:acceptor charge-transfer complex and hence an ionization efficiency of $100 \%$. However, this theoretical limit is rarely achieved because of incomplete charge transfer and the presence of unreacted dopant. Here, we establish that common p-dopants can in fact accept two electrons per molecule from conjugated polymers with a low ionization energy. Each dopant molecule participates in two charge-transfer events, leading to the formation of dopant dianions and an ionization efficiency of up to $200 \%$. Furthermore, we show that the resulting integer charge-transfer complex can dissociate with an efficiency of up to $170 \%$. The concept of double doping introduced here may allow the dopant fraction required to optimize charge conduction to be halved.
\end{abstract}

$\mathrm{R}$ edox molecular doping allows us to control the electrical properties of organic semiconductors, and is a powerful means to improve the performance of a variety of devices such as organic light-emitting diodes ${ }^{1}$, solar cells $\mathrm{s}^{2,3}$ and field-effect transistors ${ }^{4,5}$, as well as the figure of merit of thermoelectric materials ${ }^{6}$. Doping involves the addition of a molecule-a molecular dopant-to the semiconducting host material, which introduces polarons by electron transfer ${ }^{7,8}$. In the case of complete charge transfer, a cation and anion form, which, however, remain Coulombically bound to each other. At low dopant concentrations, the cation-anion pair can dissociate, leading to a free charge carrier, that is a cation (in the case of p-doping) located at a site that is sufficiently far away from the 'parent' donor site to minimize any Coulomb interaction with the anion. Instead, at high dopant concentrations the polaron is likely to be found close to one or more other anions, but nevertheless represents a hole charge carrier that can participate in transport.

Currently, for doping of polymers, redox molecular dopants are typically thought to give rise to a maximum of one charge per dopant entity in case of complete charge transfer, which corresponds to an ionization efficiency of $\eta_{\text {ion }}=100 \%$. In practice, the efficiency of redox molecular dopants is often limited by either only partial charge transfer leading to the formation of a molecular complex, which depends on the extent of overlap of the frontier orbitals and is linked to the disorder of the semiconductor ${ }^{9,10}$, or aggregation of the dopant, which reduces the number of dopant molecules that actually undergo charge transfer. Additionally, only a fraction of charges contribute significantly to transport ${ }^{9,11}$. As a result, a large amount of dopant is usually required to achieve the desired electrical conductivity ${ }^{12-14}$. Furthermore, the host material is diluted by inactive excess dopant molecules that perturb the nanostructure of the semiconductor, which tends to decrease electronic performance ${ }^{15,16}$.

A recently explored avenue for increasing the ionization efficiency is the use of dimer dopants, where one dopant moiety creates two charges, accompanied by splitting into two separate entities that act as counterions. Examples include dimeric versions of common $\mathrm{n}$-dopants based on 2,3-dihydro-1H-benzimidazole ${ }^{17}$, and dimers of organometallic sandwich compounds ${ }^{18,19}$. Since this pathway involves the dimerization of two single dopant molecules, the volume per dopant molecule that is incorporated into the semiconductor host is doubled. It would be highly desirable to identify means that permit more than one charge per dopant molecule to be created without doubling the volume, that is without dimerization.

One powerful approach to minimize the required volume/ weight fraction, and hence the effect on the nanostructure, would be to achieve transfer of more than one electron per dopant molecule, that is the formation of dopant diions. Such double doping would allow us to significantly enhance the ionization efficiency, with a maximum of $\eta_{\text {ion }}=200 \%$ if each dopant gives rise to exactly two charges. In fact, it is well known that one of the most extensively studied p-dopants, the electron acceptor 2,3,5,6-tetrafluoro-7,7,8,8tetracyanoquinodimethane (F4TCNQ), is able to form a dianion in a two-electron reduction. F4TCNQ dianions have been observed in charge-transfer salts ${ }^{20-22}$, and through photogeneration in F4TCNQ crystals $^{23,24}$. However, the current literature on molecular doping of conjugated polymers solely focuses on single-electron transfer from the host to the dopant, giving rise to (radical) monoanions. Doping studies of, for example, polythiophenes ${ }^{11,13,15,25-27}$, polyfluorenes ${ }^{27}$ and diketopyrrolopyrrole-based copolymers ${ }^{28}$, which interestingly

'Department of Chemistry and Chemical Engineering, Chalmers University of Technology, Göteborg, Sweden. ${ }^{2}$ Laboratory of Organic Electronics, Department of Science and Technology, Linköping University, Norrköping, Sweden. 'BDivision of Surface Physics and Chemistry, Department of Physics, Chemistry and Biology (IFM), Linköping University, Linköping, Sweden. ${ }^{4}$ Department of Chemistry and Centre for Plastic Electronics, Imperial College London, London, UK. Institute of Chemistry, Chemnitz University of Technology, Chemnitz, Germany. ${ }^{6}$ School of Chemistry \& Biochemistry and Center for Organic Photonics and Electronics, Georgia Institute of Technology, Atlanta, GA, USA. 'Department of Chemical Engineering, University of California Davis, Davis, CA, USA. ${ }^{8}$ Complex Materials and Devices, Department of Physics, Chemistry and Biology (IFM), Linköping University, Linköping, Sweden. ${ }^{9} \mathrm{KSC}$, King Abdullah University of Science and Technology, Thuwal, Saudi Arabia. *e-mail: kiefer@chalmers.se; christian.muller@chalmers.se 
a

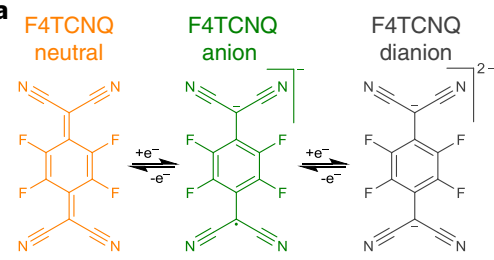

d<smiles>CC(C)(C)OCCOc1cc(-c2cc3sc(F)cc3s2)sc1-c1sc(C(C)(C)C)cc1OCCOC(C)(C)C</smiles>

g

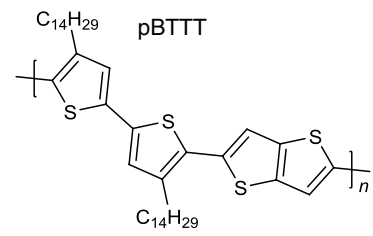
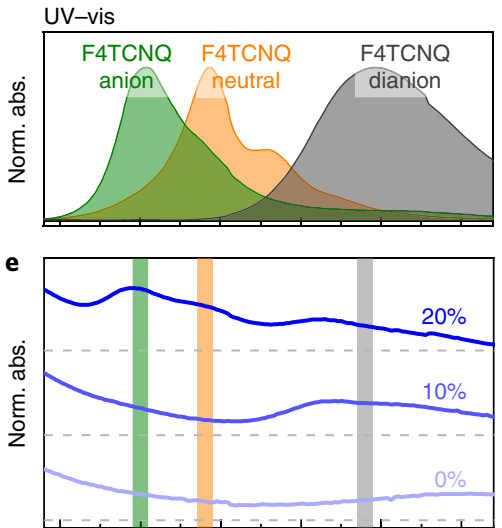

h

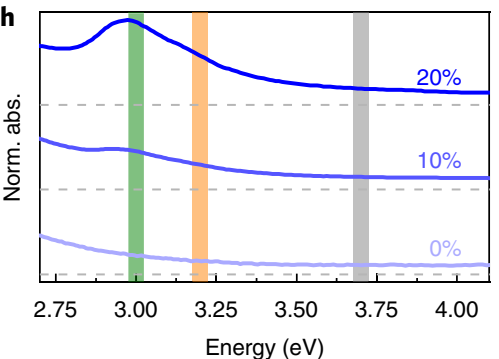

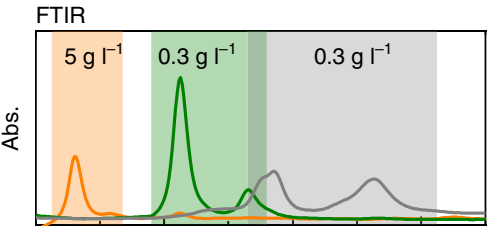
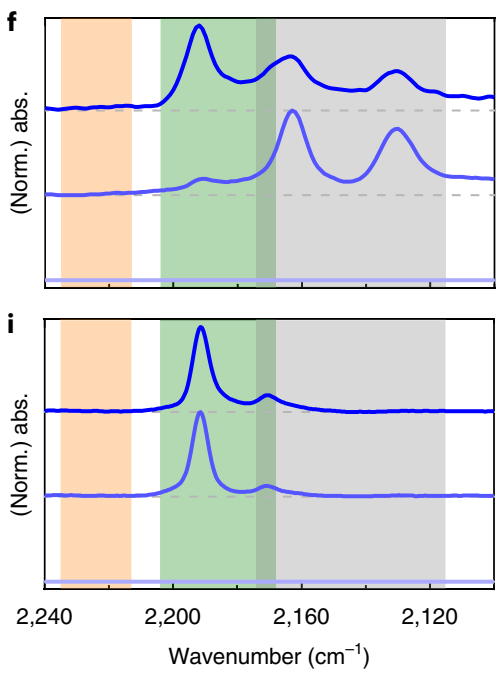

Fig. 1 | UV-vis and FTIR spectra of F4TCNQ anions and dianions. a-c, Chemical structures (a), UV-vis absorption spectra (b) and FTIR absorption (c) of the cyano stretch vibrations of neutral F4TCNQ, Li+F4TCNQ ${ }^{*-}$ and $2 \mathrm{Li}^{+} \mathrm{F} 4 \mathrm{TCNQ}{ }^{2-}$. $\mathbf{d}-\mathbf{i}$, Chemical structures of p(g $2 \mathrm{~T}-\mathrm{TT}$ ) (d) and pBTTT (g) with corresponding UV-vis absorption spectra (spectra are normalized to their highest intensity) (e,h) and FTIR absorption of the cyano stretch vibrations (f,i) of undoped films and films doped with 10 and 20 mol\% F4TCNQ (spectra of doped samples are normalized to their highest intensity). Thin films of $\mathrm{p}\left(\mathrm{g}_{4} 2 \mathrm{~T}-\mathrm{TT}\right)$ and $\mathrm{pBTTT}$ were spin coated from chloroform-acetonitrile and chlorobenzene-dichlorobenzene solutions, respectively; change of the processing solvent for $\mathrm{p}\left(\mathrm{g}_{4} 2 \mathrm{~T}-\mathrm{TT}\right)$ to chloroform or chlorobenzene resulted in comparable UV-vis and FTIR spectra (Supplementary Fig. 4).

all carry inert alkyl side chains, report the formation of not more than one charge per ionized F4TCNQ molecule. There is however no fundamental reason why F4TCNQ dianions cannot form in combination with judiciously chosen conjugated polymers.

Here, we demonstrate that common monomer molecular p-dopants such as F4TCNQ as well as the stronger acceptor $1,3,4,5,7,8$-hexafluoro-tetracyanonaphthoquinodimethane (F6TCNNQ) can abstract two electrons from conjugated polymers, leading to double doping and an ionization efficiency $\eta_{\text {ion }}$ of close to $200 \%$. We show for four different polymer:dopant pairs that the formation of dianions is favoured provided that in the low-doping regime (that is up to $10 \mathrm{~mol} \%$ dopant) the ionization energy $\left(\mathrm{IE}^{0}\right)$ of the polymer is not only less than the electron affinity of the neutral dopant $\left(\mathrm{EA}^{0}\right)$ but also less than or similar to the electron affinity of its anion $\left(\mathrm{EA}^{-}\right)$, so that both species can accept an electron from the polymer. To achieve this double doping, we selected polymers with a low $\mathrm{IE}^{0}$ of less than $5 \mathrm{eV}$, such as particular bithiophene-thienothiophene copolymers with polar oligoethylene glycol side chains, which offer excellent compatibility with molecular dopants ${ }^{12,29-31}$, but also a similar copolymer with the same backbone that carries non-polar alkoxy side chains. The double-doping concept considerably extends the versatility of this emerging class of polar conjugated polymers, which currently receive attention as the electrode material for batteries ${ }^{32,33}$, as donor and acceptor materials for organic solar cells $^{34}$, as the active layer of $\mathrm{p}$ - and n-type electrochemical transistors $^{35-37}$ and as $\mathrm{p}$ - and n-type conductors for thermoelectrics ${ }^{12,29,30}$.

We chose to focus our study on a bithiophene-thienothiophene copolymer with tetraethylene glycol side chains, $\mathrm{p}\left(\mathrm{g}_{4} 2 \mathrm{~T}-\mathrm{TT}\right)$ (see Fig. 1 for chemical structure). Doping of $\mathrm{p}\left(\mathrm{g}_{4} 2 \mathrm{~T}-\mathrm{TT}\right)$ with only $10 \mathrm{~mol} \%$ ( $4 \mathrm{wt} \%$ ) of F4TCNQ through coprocessing from a chloroform-acetonitrile solution gives rise to a relatively high conductivity of $2 \mathrm{~S} \mathrm{~cm}^{-1}$, which is similar to values reported for the comparable polymer with alkyl side chains, that is the widely studied polymer pBTTT (Fig. 1), when coprocessed with $25 \mathrm{~mol} \%$ F4TCNQ $^{38}$. Surprisingly, the ultraviolet-visible (UV-vis) absorption spectrum of doped $\mathrm{p}\left(\mathrm{g}_{4} 2 \mathrm{~T}-\mathrm{TT}\right)$ does not show the typical F4TCNQ anion absorption peaks at about $768 \mathrm{~nm}$ and about $864 \mathrm{~nm}$, located below the bandgap of the polymer (Supplementary Fig. 2). The apparent absence of anions, in the presence of the polymer polaron peak, suggests that dianions, which do not absorb at visible wavelengths, are present instead (Supplementary Fig. 3).

In order to establish a means to quantify the amount of neutral, anionic and dianionic F4TCNQ, we set out to determine the molar absorption coefficients of all three species. We synthesized the mono- $\left(\mathrm{Li}^{+} \mathrm{F} 4 \mathrm{TCNQ}{ }^{--}\right)$and dilithium $\left(2 \mathrm{Li}^{+} \mathrm{F} 4 \mathrm{TCNQ}^{2-}\right)$ salts of F4TCNQ and recorded UV-vis absorption spectra, which agree with literature (Fig. 1 and Supplementary Fig. 3) ${ }^{22}$. Deconvolution of the absorption spectra of F4TCNQ-doped semiconductors regarding the signature peaks of neat F4TCNQ and its anion and dianion between 2.7 and $4.1 \mathrm{eV}$ provides a first means for a qualitative analysis of the abundance of the three species in doped films. However, due to strong differences of their molar absorption coefficients (Supplementary Fig. 3) we chose to complement UV-vis absorption measurements with transmission Fourier transform infrared (FTIR) spectroscopy. We recorded the stretching frequencies of the cyano groups $(\mathrm{CN})$ in F4TCNQ, whose absorption energies are indicative of charge transfer, and find a weak single peak at $2,228 \mathrm{~cm}^{-1}$ for neat F4TCNQ (meaning that the amount of neutral dopant cannot be quantified with FTIR). Instead, for $\mathrm{Li}^{+} \mathrm{F} 4 \mathrm{TCNQ}{ }^{--}$the $\mathrm{CN}$ vibrations shift to $2,195 \mathrm{~cm}^{-1}$ accompanied by an increase of the oscillator strength and a second peak at $2,174 \mathrm{~cm}^{-1}$, followed by a further shift to $2,166 \mathrm{~cm}^{-1}$ and $2,135 \mathrm{~cm}^{-1}$ for $2 \mathrm{Li}^{+} \mathrm{F} 4 \mathrm{TCNQ}{ }^{2-}$ (Fig. 1c). 

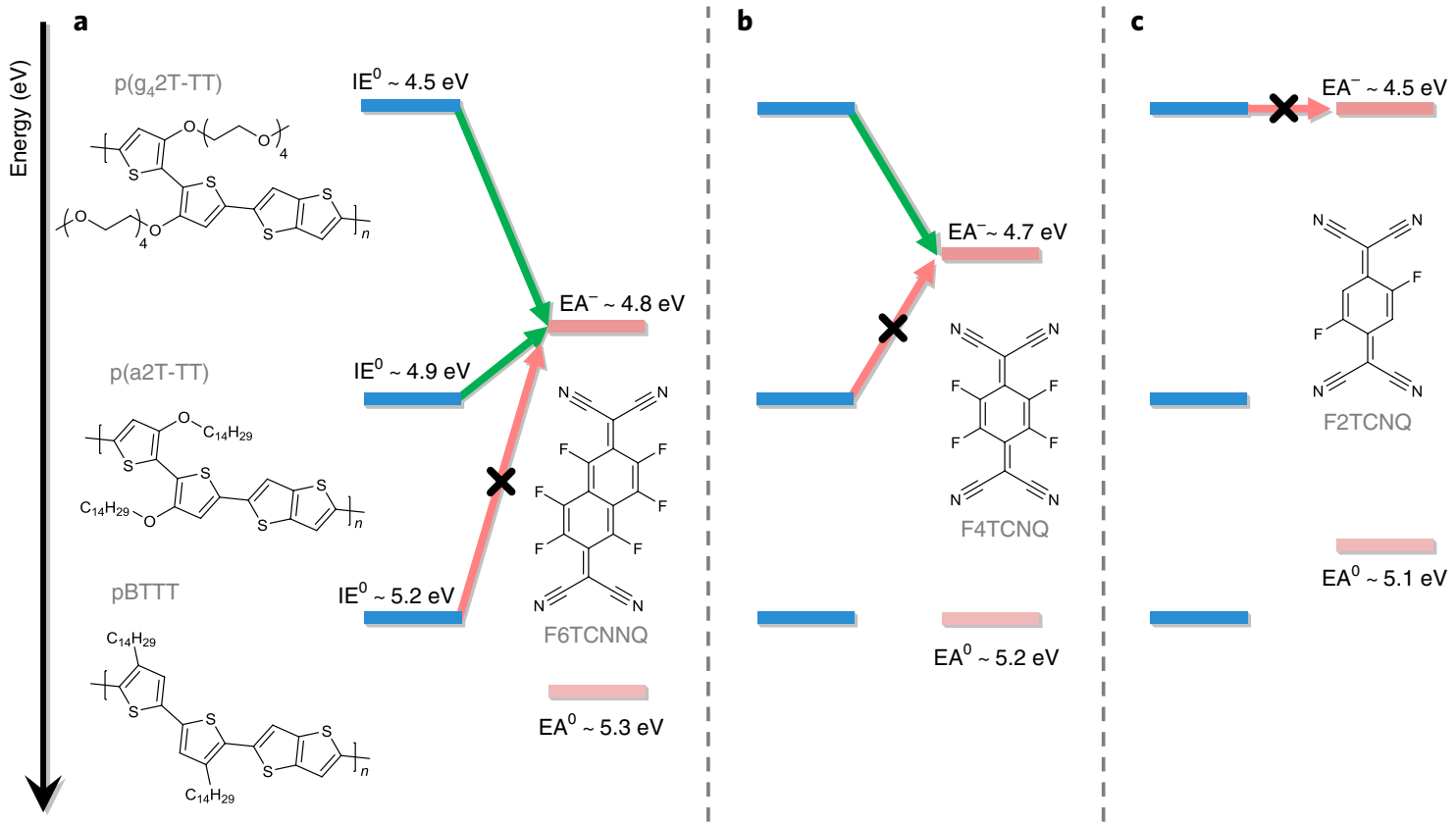

Fig. 2 | Energy diagram summarizing the formation of dopant dianions. a-c, Doping of $p\left(g_{4} 2 T-T T\right), p(a 2 T-T T)$ and pBTTT with F6TCNNQ (a), F4TCNQ (b) and F2TCNQ (c); IE $\mathrm{E}^{0}$ of the polymers as well as $\mathrm{EA}^{\circ}$ and $\mathrm{EA}^{-}$of the dopants were measured with cyclic voltammetry (Supplementary Figs. 5 and 6 ). Green (red) arrows indicate where electron transfer from the polymer to the dopant anion is observed (absent).

The shifts and the peak intensities that correspond to experimental and calculated values reported for neutral F4TCNQ and its anion and dianion ${ }^{22,39}$ allow us to confirm the relative numbers of anions and dianions by FTIR. We carried out a similar analysis to investigate the presence of dianions of the dopants 2,5-difluoro-7,7,8,8tetracyanoquinodimethane (F2TCNQ) and F6TCNNQ.

We then chose to study the influence of the ionization energy of the polymer. A comparison of UV-vis and FTIR spectra indicates that a large fraction of dianions is present in $\mathrm{p}\left(\mathrm{g}_{4} 2 \mathrm{~T}-\mathrm{TT}\right)$, whereas in pBTTT only anions can be discerned (Fig. 1). We argue that electron transfer (that is ion pair formation) is aided by a favourable offset between the $\mathrm{IE}^{0}$ of the donor and the electron affinity of the acceptor, where we must distinguish between the $\mathrm{EA}^{0}$ of the neutral dopant and the $\mathrm{EA}^{-}$of the anion. We find that the $\mathrm{IE}^{0} \sim 4.5 \mathrm{eV}$ of $\mathrm{p}\left(\mathrm{g}_{4} 2 \mathrm{~T}-\mathrm{TT}\right.$ ) (Supplementary Fig. 5) is considerably smaller than the $\mathrm{IE}^{0} \sim 5.2 \mathrm{eV}$ of pBTTT. Hence, neutral pBTTT is able to donate an electron to neutral F4TCNQ with $\mathrm{EA}^{0} \sim 5.2 \mathrm{eV}$, but cannot donate a further electron to its corresponding anion since $\mathrm{EA}^{-} \ll \mathrm{IE}^{0}$, which rules out the formation of dianions. In contrast, in the case of $\mathrm{p}\left(\mathrm{g}_{4} 2 \mathrm{~T}-\mathrm{TT}\right)$ the $\mathrm{IE}^{0}$ is less than both the $\mathrm{EA}^{0}$ of neutral F4TCNQ and the $\mathrm{EA}^{-}$of its anion, leading to efficient formation of the dianion.

To further test the importance of the ionization energy of the polymer, we included a third polymer in our analysis, p(a2T-TT) (Fig. 2), which has an $\mathrm{IE}^{0} \sim 4.9 \mathrm{eV}$. Furthermore, we exchanged F4TCNQ with either the weaker dopant F2TCNQ or the stronger dopant F6TCNNQ. Doping of $\mathrm{p}\left(\mathrm{g}_{4} 2 \mathrm{~T}-\mathrm{TT}\right)$ with F2TCNQ, where $\mathrm{EA}^{-} \sim \mathrm{IE}^{0}$, only yields anions, whereas doping with F6TCNNQ readily results in dianion formation since $\mathrm{EA}^{-} \ll \mathrm{IE}^{0}$ (Supplementary Figs. 7 and 8), which confirms the importance of a sufficiently low $\mathrm{IE}^{0}$. Instead, for doping of $\mathrm{p}(\mathrm{a} 2 \mathrm{~T}-\mathrm{TT})$ with any of the three dopants the energy offset is unfavourable for dianion formation since $\mathrm{EA}^{-}<\mathrm{IE}^{0}$. We indeed only observe anions when doping $\mathrm{p}(\mathrm{a} 2 \mathrm{~T}$ TT) with F4TCNQ (Supplementary Fig. 9). In contrast, doping of $\mathrm{p}(\mathrm{a} 2 \mathrm{~T}-\mathrm{TT})$ with the stronger acceptor F6TCNNQ readily yields dianions although $\mathrm{EA}^{-}<\mathrm{IE}^{0}$ by $0.1 \mathrm{eV}$ (Supplementary Fig. 9). We argue that dianion formation is determined not only by the offset between $\mathrm{IE}^{0}$ and $\mathrm{EA}^{-}$but also by electrostatic interactions as shown for pentacene:F4TCNQ ${ }^{40}$. Analogously, anion formation through complete charge transfer has been reported for polymer:dopant systems such as pBTTT:F2TCNQ despite an unfavourable energy offset, that is $\mathrm{EA}^{0}<\mathrm{IE}^{0}$ (ref. ${ }^{41,42}$ ).

We would like to draw attention to the different polarities of $\mathrm{p}\left(\mathrm{g}_{4} 2 \mathrm{~T}-\mathrm{TT}\right)$ and $\mathrm{p}(\mathrm{a} 2 \mathrm{~T}-\mathrm{TT})$. The tetraethylene glycol side chains of $\mathrm{p}\left(\mathrm{g}_{4}\right.$ 2T-TT) lead to a dielectric constant of $\varepsilon_{\mathrm{r}} \sim 4.2$, higher than that of pBTTT with $\varepsilon_{\mathrm{r}} \sim 2.6$ (Supplementary Fig. 10). For p(a2T-TT) we expect a dielectric constant similar to that of pBTTT. Since we observe dianion formation in the case of $\mathrm{p}(\mathrm{a} 2 \mathrm{~T}-\mathrm{TT})$ :F6TCNNQ but not $\mathrm{p}\left(\mathrm{g}_{4} 2 \mathrm{~T}-\mathrm{TT}\right)$ :F2TCNQ despite an unfavourable energy offset in both cases, we conclude that a high $\varepsilon_{\mathrm{r}}$ and therefore greater dielectric background screening is not required for formation and stabilization of the dianion.

In a further set of experiments we investigated whether the location of the dopant in the polymer matrix or the polymer constitution influence double doping. A combination of grazing-incidence wide-angle X-ray scattering (GIWAXS), density functional theory (DFT) calculations and FTIR spectroscopy indicates that the dopant is incorporated between the side chains of the polymer, with an approximate donor:acceptor distance of about $6.5 \AA$. We argue that in ordered but also in amorphous domains the dopant prefers to reside within the tetraethylene glycol side chains, and that dianion formation readily occurs in both cases (see Supplementary Information, Section 8). To probe the influence of the polymer constitution we compared doping of three batches of $\mathrm{p}\left(\mathrm{g}_{4} 2 \mathrm{~T}-\mathrm{TT}\right)$ with different molecular weights, obtained by fractionation, as well as a fourth polymer with a low $\mathrm{IE}^{0}$ that we added to our study, $\mathrm{p}\left(\mathrm{g}_{3} 2 \mathrm{~T}\right.$ TT), which carries shorter triethylene glycol side chains. FTIR spectroscopy indicates that the extent of double doping depends on both the molecular weight and the side chain length. We conclude that double doping could be further optimized by adjusting these parameters, provided that processing issues can be overcome (see Supplementary Information, Section 9). 

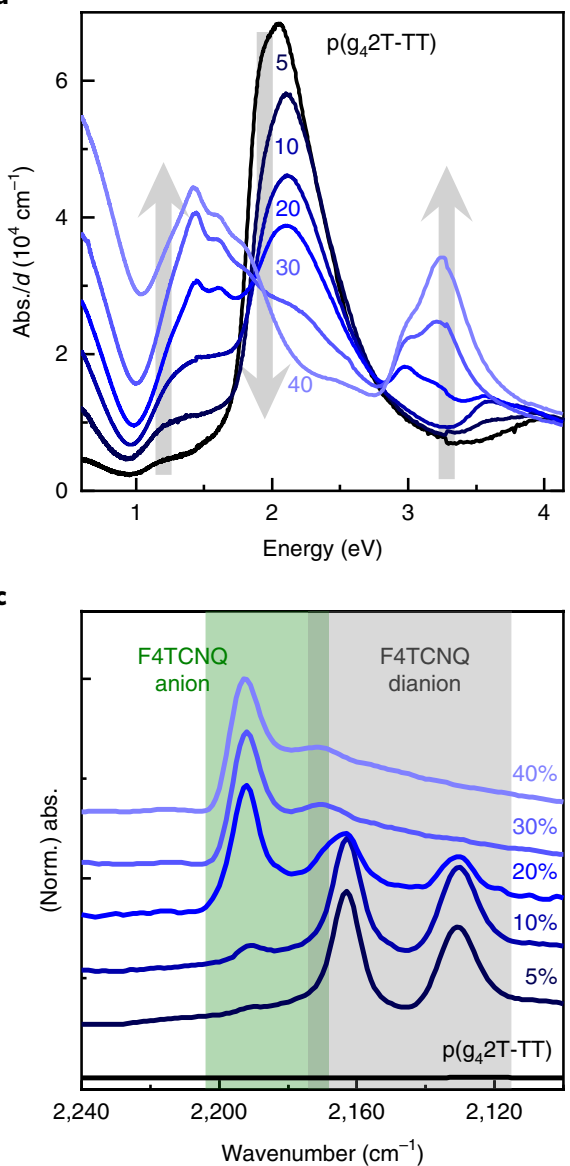

b

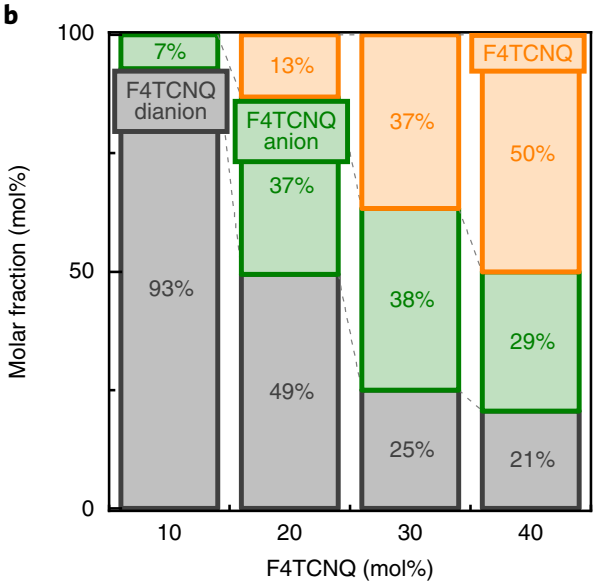

d

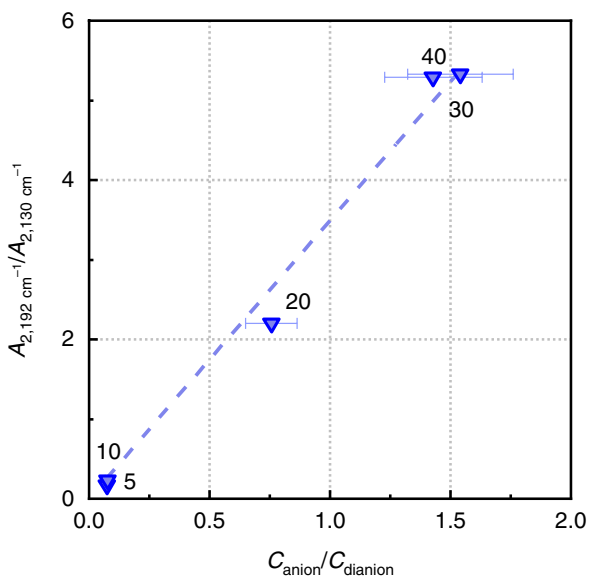

Fig. 3 | Amount of neutral, anionic and dianionic F4TCNQ in doped $\mathrm{p}\left(\mathrm{g}_{4} \mathbf{2 T}-\mathrm{TT}\right.$ ) films. a, Normalized UV-vis absorption spectra of pristine and doped (5, $10,20,30,40 \mathrm{~mol} \%$ per repeat unit) $\mathrm{p}\left(\mathrm{g}_{4} 2 \mathrm{~T}-\mathrm{TT}\right)$ films (thickness, $\left.d=50-250 \mathrm{~nm}\right)$. b. The corresponding molar fractions of neutral F4TCNQ, anion and dianion derived by fitting of the absorption coefficients (see Supplementary Figs. 11 and 12). c, Solid-state FTIR absorption spectra of pristine $\mathrm{p}\left(\mathrm{g}_{4} 2 \mathrm{2T}-\mathrm{TT}\right.$ ) and $\mathrm{p}\left(\mathrm{g}_{4} 2 \mathrm{~T}-\mathrm{TT}\right)$ doped with 5-40 mol\% F4TCNQ. d, Linear correlation between the relative FTIR absorption intensity at $2,192 \mathrm{~cm}^{-1}(\mathrm{anion})$ and $2,130 \mathrm{~cm}^{-1}$ (dianion) and the relative concentrations of F4TCNQ anion and dianion; error bars represent the estimated error of the applied fitting procedure (the dashed line is a linear fit to the data).

We now turn our attention to the influence of the dopant fraction on dianion formation in $\mathrm{p}\left(\mathrm{g}_{4} 2 \mathrm{~T}-\mathrm{TT}\right)$. We recorded a set of UVvis spectra of both polymer:dopant solutions and spin-coated films, and used these to quantify the different species of F4TCNQ (Fig. 3; see Methods for details). Furthermore, we used FTIR to confirm the ratio of anions and dianions determined with UV-vis. We find that in films doped with $10 \mathrm{~mol} \%$ F4TCNQ all dopant molecules have undergone charge transfer with $\mathrm{p}\left(\mathrm{g}_{4} 2 \mathrm{~T}-\mathrm{TT}\right)$, with more than $90 \%$ being present as dianions (Fig. $3 \mathrm{~b}$ ), which implies that doping occurs with an efficiency of $\eta_{\text {ion }}>190 \%$. The absolute concentration of dianions $\left(\sim 10^{-4} \mathrm{~mol} \mathrm{~cm}^{-3}\right.$; Supplementary Fig. 23) remains almost unchanged upon doping with more than $10 \mathrm{~mol} \%$ F4TCNQ, but is accompanied by an increasing number of anions and later neat F4TCNQ (cf. Figure 3). We explain this trend by the relatively high charge density of one positive charge per approximately five polymer repeat units already for $10 \mathrm{~mol} \%$ (Supplementary Fig. 24), meaning that only a few undoped regions remain that are large enough to accommodate two polarons and hence allow further double doping. The charge density further increases to one charge per approximately three repeat units for $30 \mathrm{~mol} \%$, above which the anion concentration also levels off (Supplementary Fig. 23).

To gain further evidence for dianion formation, and to discern its impact on the electrical properties, we explored doping of $\mathrm{p}\left(\mathrm{g}_{4} 2 \mathrm{~T}-\mathrm{TT}\right)$ directly with the F4TCNQ anion. We dissolved varying amounts of $\mathrm{Li}^{+} \mathrm{F} 4 \mathrm{TCNQ}{ }^{\bullet-}$ together with the polymer, cast films and recorded FTIR absorption spectra of the cyano stretch vibrations (Fig. 4a). F4TCNQ dianions readily form and are present to almost $100 \%$ in the case of $10 \mathrm{~mol} \% \mathrm{Li}^{+} \mathrm{F}_{4} \mathrm{TCNQ}^{--}$(Fig. 4b). Despite a gradual decrease in the dianion to anion ratio, even for $40 \mathrm{~mol} \%$ $\mathrm{Li}^{+} \mathrm{F} 4 \mathrm{TCNQ}{ }^{--}$we still observe a majority of dianions. $\mathrm{Li}^{+} \mathrm{F} 4 \mathrm{TCNQ}{ }^{\bullet-}$ can only accept one electron from $\mathrm{p}\left(\mathrm{g}_{4} 2 \mathrm{~T}-\mathrm{TT}\right)$ and therefore only exhibits a maximum ionization efficiency of one charge per dopant (Fig. 4c). Instead, doping of $\mathrm{p}\left(\mathrm{g}_{4} 2 \mathrm{~T}-\mathrm{TT}\right)$ with neat F4TCNQ can give rise to up to two charges per dopant molecule (Fig. 4c), which should have a noticeable effect on the electrical properties.

We measured the electrical conductivity of thin films (thickness $50-250 \mathrm{~nm}$ ) of $\mathrm{p}\left(\mathrm{g}_{4} 2 \mathrm{~T}-\mathrm{TT}\right)$ doped by coprocessing (Fig. $4 \mathrm{~d}$ and Supplementary Fig. 25). Doping with only $10 \mathrm{~mol} \%$ F4TCNQ $(\sim 4 \mathrm{wt} \%)$ results in an electrical conductivity of $\sigma \sim 2 \mathrm{~S} \mathrm{~cm}^{-1}$. The conductivity continuously increases by about two orders of magnitude to a maximum conductivity of $\sigma \sim 100 \mathrm{~S} \mathrm{~cm}^{-1}$ for $40 \mathrm{~mol} \%$ (note that we obtain a similar value for vapour-doped samples; see Supplementary Fig. 26), until it drops by about one order of magnitude for a dopant fraction of $50 \mathrm{~mol} \%$, probably due to disruption of the nanostructure. In contrast, addition of $\mathrm{Li}^{+} \mathrm{F} 4 \mathrm{TCNQ}{ }^{--}$gives rise to a consistently lower electrical conductivity, which increases from $1 \mathrm{Scm}^{-1}$ for $10 \mathrm{~mol} \%$ to $18 \mathrm{Scm}^{-1}$ for $40 \mathrm{~mol} \%$. At the same time, we find that $\mathrm{p}\left(\mathrm{g}_{4} 2 \mathrm{~T}-\mathrm{TT}\right)$ doped with F4TCNQ displays a 
a

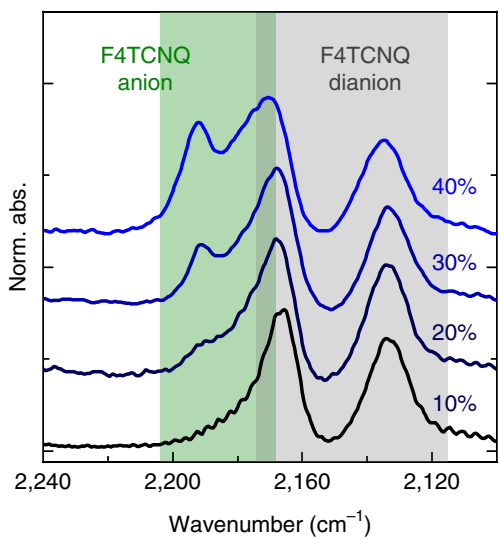

c

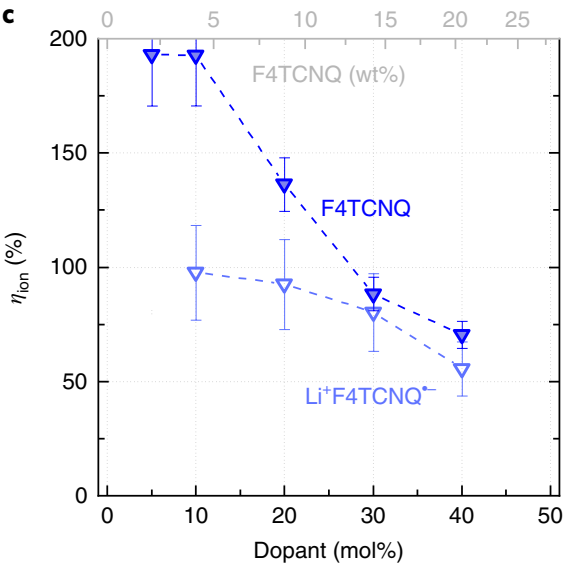

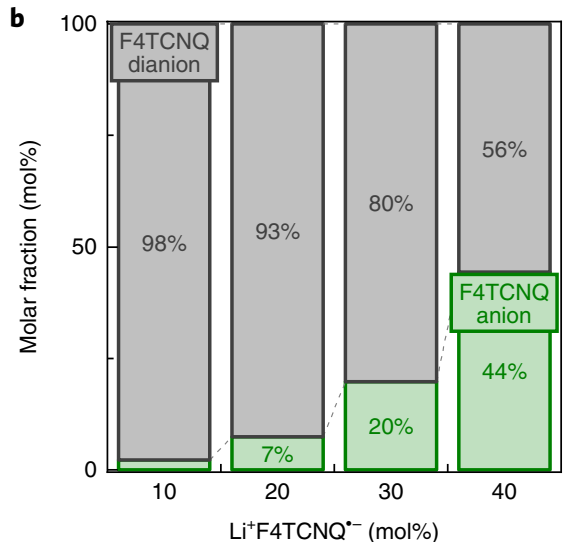

d

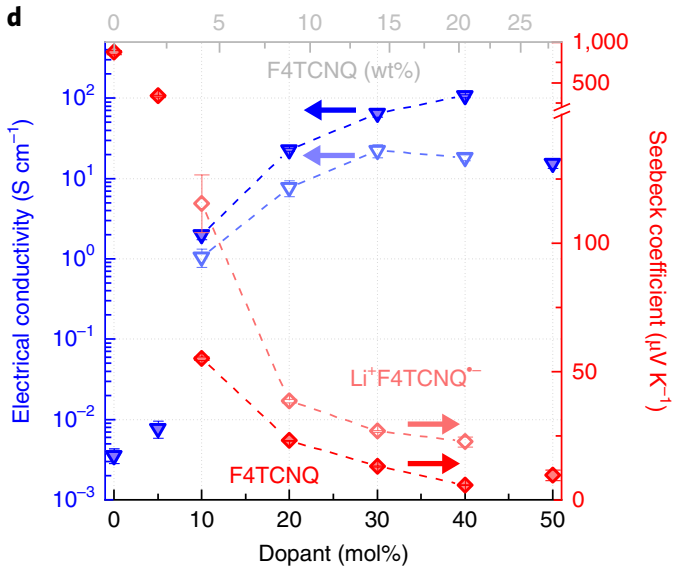

Fig. 4 | Comparison of doping of $\mathbf{p}\left(\mathrm{g}_{4}\right.$ 2T-TT) with F4TCNQ and Li+F4TCNQ•-. a, Solid-state FTIR absorption spectra of $p\left(\mathrm{~g}_{4} 2 \mathrm{~T}-\mathrm{TT}\right)$ doped with 10, 20, 30 and $40 \mathrm{~mol} \% \mathrm{Li}^{+} \mathrm{F} 4 \mathrm{TCNQ}{ }^{--}$per repeat unit, and $\mathbf{b}$, molar fractions of F4TCNQ anion and dianion in the corresponding films determined from the relative FTIR intensities of anions $\left(2,192 \mathrm{~cm}^{-1}\right)$ and dianions $\left(2,131 \mathrm{~cm}^{-1}\right.$; cf. Fig. $\left.3 \mathrm{~d}\right)$ ). c, lonization efficiency, $\eta_{\text {ion }}$ (error bars represent the estimated error of the applied fitting procedure), and $\mathbf{d}$, electrical conductivity and Seebeck coefficient of $\mathrm{p}\left(\mathrm{g}_{4} 2 \mathrm{~T}-\mathrm{TT}\right)$ doped with various amounts of F4TCNQ (open symbols) and $\mathrm{Li}^{+} \mathrm{F} 4 \mathrm{TCNQ}{ }^{--}$(closed symbols); error bars represent the standard deviation of five measurements on the same sample (dashed lines are guides to the eye).

significantly lower Seebeck coefficient as compared with samples doped with $\mathrm{Li}^{+} \mathrm{F} 4 \mathrm{TCNQ}{ }^{\bullet-}$, for example $\alpha \sim 55$ and $115 \mu \mathrm{V} \mathrm{K}^{-1}$ in the case of $10 \mathrm{~mol} \%$ dopant, which is indicative of a lower chargecarrier concentration in the $\mathrm{Li}^{+} \mathrm{F} 4 \mathrm{TCNQ}{ }^{\bullet-}$ doped material (Fig. 4d). We rule out the possibility that the difference in thermoelectric properties arises due to differences in nanostructure, as confirmed by GIWAXS (Supplementary Figs. 15 and 16). For both dopants, the Seebeck coefficient and electrical conductivity roughly scale according to the often-observed empirical relationship $\alpha \propto \sigma^{-0.25}$ (Supplementary Fig. 27). Since the number of mobile charges correlates with the Seebeck coefficient, we can compare the number of holes that contribute to charge transport. For a doping fraction of $10 \mathrm{~mol} \%$ we find that doping of $\mathrm{p}\left(\mathrm{g}_{4} 2 \mathrm{~T}-\mathrm{TT}\right)$ with F4TCNQ gives rise to twice as many mobile charges as doping with $\mathrm{Li}^{+} \mathrm{F} 4 \mathrm{TCNQ}{ }^{-}$ (Supplementary Information, Section 13).

The high concentration of polarons in F4TCNQ-doped $\mathrm{p}\left(\mathrm{g}_{4} 2 \mathrm{~T}\right.$ TT) was furthermore confirmed by ultraviolet photoelectron spectroscopy (UPS) (Fig. 5a,b). With increasing dopant fraction, we observed a significant increase of the work function from $4.15 \mathrm{eV}$ to $4.70 \mathrm{eV}$ along with a shift of the Fermi energy $\left(E_{\mathrm{F}}\right)$ towards the valence band edge (VBE), which is characteristic for p-type doping. We observe a delayed shift of the VBE with increasing F4TCNQ content, which we assign to oxygen doping of the polymer (cf. Supplementary Fig. 25). For dopant fractions of more than $10 \mathrm{~mol} \%$ the valence region of the spectrum is broadened and the onset of the spectrum coincides with $E_{\mathrm{F}}$ which reflects the broadening of the density of states in the vicinity of the VBE upon doping.
To estimate the dissociation efficiency at a low dopant fraction of $0.5 \mathrm{~mol} \%$ dopant, we constructed Mott-Schottky diodes, commonly used to determine the density of contributing charges in doped semiconductors $^{9}$. We deduce a dissociation efficiency $\eta_{\text {diss }} \sim 172 \%$ for $\mathrm{p}\left(\mathrm{g}_{4} 2 \mathrm{~T}-\mathrm{TT}\right)$ doped with F4TCNQ (Supplementary Fig. 28), that is, each dopant molecule gives rise to close to two free charges. For $\mathrm{p}\left(\mathrm{g}_{4} 2 \mathrm{~T}-\mathrm{TT}\right)$ doped with $\mathrm{Li}^{+} \mathrm{F} 4 \mathrm{TCNQ}{ }^{--}$we instead observe a significantly lower $\eta_{\text {diss }} \sim 76 \%$, which confirms that double doping indeed gives rise to twice the number of free charges.

To gain insight into the fraction of charges that contribute to transport at higher dopant concentration, we paired variable-temperature conductivity measurements with kinetic Monte Carlo modelling. Independent of the dopant fraction, $\mathrm{p}\left(\mathrm{g}_{4} 2 \mathrm{~T}-\mathrm{TT}\right)$ doped with F4TCNQ shows a typical semiconducting behaviour of thermally activated charge transport (Fig. 5c), which is characteristic for disordered conducting polymers ${ }^{43}$. We used kinetic Monte Carlo modelling described previously for poly(3-hexylthiophene):F4TCNQ ${ }^{44}$ to extract the density of states and the distribution of hole charge carriers (density of occupied states, DOOS; Supplementary Fig. 29). The model considers nearest-neighbour hopping on a cubic lattice with lattice constant $a_{\mathrm{NN}}=1 \mathrm{~nm}$. The energy of each site is randomly sampled from a Gaussian density of states (initial disorder $75 \mathrm{meV}$ ) that is adjusted for Coulomb interactions with all electrons, cations and (di)anions. In the high-doping regime (20 mol\% F4TCNQ) the simulations describe the variable-temperature conductivity measurements well (Fig. 5c). At lower doping fractions the simulated conductivity is overestimated, which we explain by the heterogeneous 


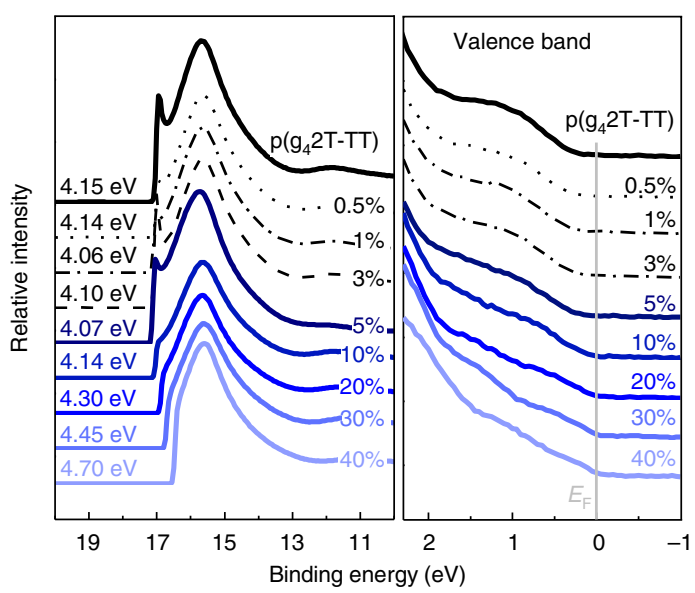

b

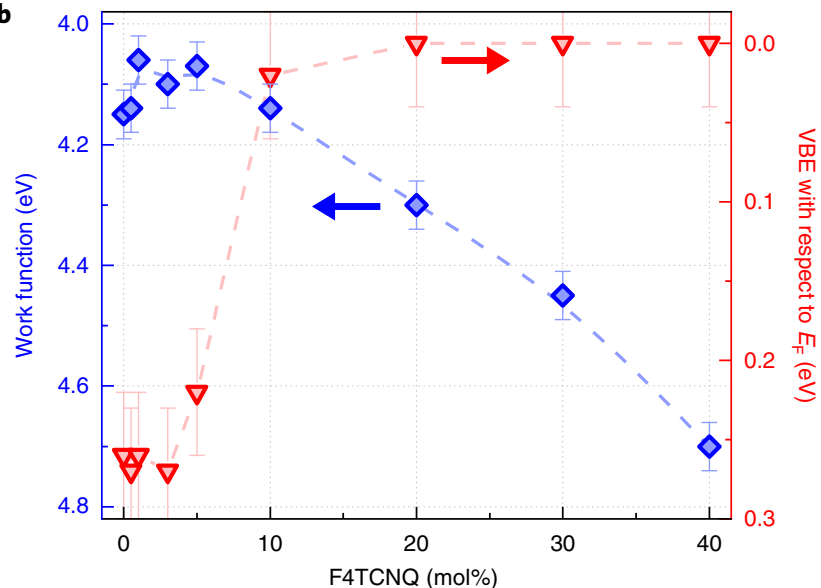

c

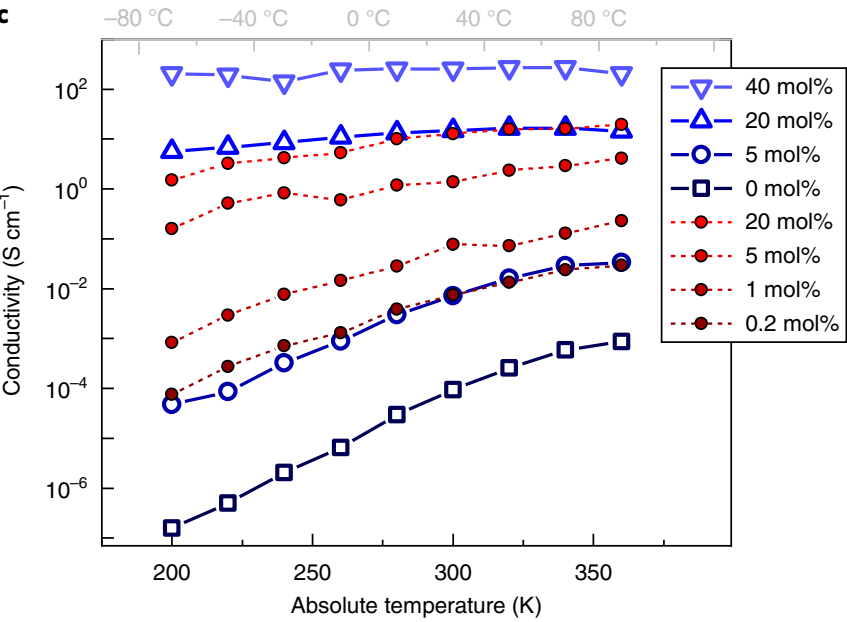

Fig. $\mathbf{5}$ | Work function and temperature-dependent conductivity of F4TCNQ doped $p\left(g_{4} 2 T-T T\right)$. a, UPS spectra of $p\left(g_{4} 2 T-T T\right)$ neat and doped with increasing amounts of F4TCNQ showing the photoemission cut-off (left) and the valence band region (right). b. Work function and VBE with respect to $E_{\mathrm{F}}$ of $\mathrm{p}\left(\mathrm{g}_{4} 2 \mathrm{~T}-\mathrm{TT}\right)$ doped with $\mathrm{F} 4 \mathrm{TCNQ}$; error bars represent the total energy resolution of the UPS measurements. c, Experimental temperature-dependent conductivity measurements of $p\left(g_{4} 2 T-T T\right)$ neat and doped with 5, 20 and 40 mol\% F4TCNQ (open symbols), and simulated temperature-dependent conductivity for $0.2,1,5$ and $20 \mathrm{~mol} \%$ F4TCNQ using kinetic Monte Carlo modelling (red circles). Experimental data points in c were derived by linear fitting of current-voltage curves with standard deviations smaller than $1 \%$. Simulated conductivity values are within $\pm 10-20 \%$ error (lines in $\mathbf{b}$ and $\mathbf{c}$ are guides to the eye). nanostructure of $\mathrm{p}\left(\mathrm{g}_{4} 2 \mathrm{~T}-\mathrm{TT}\right)$ consisting of amorphous and ordered domains, which is not captured by our model. We estimate the fraction of charges that contribute to transport, $f_{\text {trans }}$, by integrating the DOOS between $E_{\mathrm{F}} \pm 2 k_{\mathrm{B}} T$, and dividing by the total DOOS. For $\mathrm{p}\left(\mathrm{g}_{4} 2 \mathrm{~T}-\mathrm{TT}\right)$ doped with $20 \mathrm{~mol} \%$ F4TCNQ we obtain a value of $f_{\text {trans }}=5 \%$, which increases to about $30 \%$ at lower dopant concentrations (Supplementary Fig. 30a). Note that $f_{\text {trans }}$ is the fraction of charges that contribute to transport at an arbitrary point in time; when averaged over time all charges contribute to transport. As argued above, for the high dopant concentrations explored here, the majority of charges are not free since statistically few sites remain that are sufficiently far away from an anion to not feel a (screened) Coulomb interaction. Interestingly, our kinetic Monte Carlo calculations yield an ionization efficiency of at least $\eta_{\text {ion }} \sim 150 \%$ for $20 \mathrm{~mol} \%$ of F4TCNQ (see Supplementary Figure 29), in agreement with our experimentally obtained values (cf. Figure $4 \mathrm{c}$ ). For $\mathrm{p}\left(\mathrm{g}_{4} 2 \mathrm{~T}\right.$ TT) doped with $10 \mathrm{~mol} \% \mathrm{Li}^{+} \mathrm{F} 4 \mathrm{TCNQ}^{\bullet-}$, in contrast, we calculate a much lower DOOS as compared with doping with F4TCNQ (Supplementary Fig. 31), which implies a significantly lower $\eta_{\text {ion }} \sim 92 \%$. Since $f_{\text {trans }}$ values are similar for the two dopants $(13 \%$ and $17 \%$ ), we conclude that double doping considerably increases the number of charges that contribute to transport also at higher dopant concentration.

Finally, our UV-vis analysis allows us to estimate the density of all bound plus mobile charges by counting the total number of electrons accepted by all anions and dianions. We estimate a value of 2 to $4 \times 10^{20}$ charges $\mathrm{cm}^{-3}$, which yields a lower bound for the charge-carrier mobility of $\mu \sim 0.06-2 \mathrm{~cm}^{2} \mathrm{~V}^{-1} \mathrm{~s}^{-1}$ for an F4TCNQ fraction ranging from 10 to $40 \mathrm{~mol} \%$ (Supplementary Fig. 30b). Thus, we can assume that the electrical conductivity of a highmobility polymer could be greatly enhanced by taking advantage of dianion formation.

In summary, we have established that double doping of conjugated polymers can be carried out with common p-dopants, leading to an ionization efficiency of up to $200 \%$. Dianion formation is found to occur for four different polymer:dopant pairs, which suggests that double doping is a generic principle that applies to a wide range of organic semiconductors with appropriate energy levels. We predict that the design of polymers with higher charge-carrier mobility and energy levels specifically devised for applications such as thermoelectrics or bioelectronics, and the use of stronger dopants with the ability to abstract two electrons from polymers with a higher $\mathrm{IE}^{0}$, will lead to a wide class of highly conducting materials that harness double doping.

\section{Online content}

Any methods, additional references, Nature Research reporting summaries, source data, statements of data availability and associated accession codes are available at https://doi.org/10.1038/ s41563-018-0263-6.

Received: 28 May 2018; Accepted: 23 November 2018; Published online: 14 January 2019

\section{References}

1. Walzer, K., Maennig, B., Pfeiffer, M. \& Leo, K. Highly efficient organic devices based on electrically doped transport layers. Chem. Rev. 107, 1233-1271 (2007)

2. Lu, L. et al. Recent advances in bulk heterojunction polymer solar cells. Chem. Rev. 115, 12666-12731 (2015).

3. Salzmann, I. \& Heimel, G. Toward a comprehensive understanding of molecular doping organic semiconductors (review). J. Electron Spectrosc. Relat. Phenom. 204, 208-222 (2015).

4. Lüssem, B. et al. Doped organic transistors. Chem. Rev. 116, 13714-13751 (2016).

5. Lu, G. et al. Moderate doping leads to high performance of semiconductor/ insulator polymer blend transistors. Nat. Commun. 4, 1588 (2013). 
6. Russ, B., Glaudell, A., Urban, J. J., Chabinyc, M. L. \& Segalman, R. A. Organic thermoelectric materials for energy harvesting and temperature control. Nat. Rev. Mater. 1, 16050 (2016).

7. Jacobs, I. E. \& Moule, A. J. Controlling molecular doping in organic semiconductors. Adv. Mater. 29, 1703063 (2017).

8. Kroon, R. et al. Thermoelectric plastics: from design to synthesis, processing and structure-property relationships. Chem. Soc. Rev. 45, 6147-6164 (2016).

9. Tietze, M. L. et al. Elementary steps in electrical doping of organic semiconductors. Nat. Commun. 9, 1182 (2018).

10. Jacobs, I. E. et al. Polymorphism controls the degree of charge transfer in a molecularly doped semiconducting polymer. Mater. Horiz. 5, 655-660 (2018).

11. Pingel, P. \& Neher, D. Comprehensive picture of p-type doping of P3HT with the molecular acceptor F4TCNQ. Phys. Rev. B 87, 115209 (2013).

12. Kiefer, D. et al. Enhanced n-doping efficiency of a naphthalenediimide-based copolymer through polar side chains for organic thermoelectrics. ACS Energy Lett. 3, 278-285 (2018).

13. Jacobs, I. E. et al. Comparison of solution-mixed and sequentially processed P3HT:F4TCNQ films: effect of doping-induced aggregation on film morphology. J. Mater. Chem. C 4, 3454-3466 (2016).

14. Euvrard, J. et al. The formation of polymer-dopant aggregates as a possible origin of limited doping efficiency at high dopant concentration. Org. Electron. 53, 135-140 (2018).

15. Duong, D. T., Wang, C. C., Antono, E., Toney, M. F. \& Salleo, A. The chemical and structural origin of efficient p-type doping in P3HT. Org. Electron. 14, 1330-1336 (2013).

16. Schlitz, R. A. et al. Solubility-limited extrinsic n-type doping of a high electron mobility polymer for thermoelectric applications. Adv. Mater. 26, 2825-2830 (2014).

17. Naab, B. D. et al. Effective solution- and vacuum-processed n-doping by dimers of benzimidazoline radicals. Adv. Mater. 26, 4268-4272 (2014).

18. Perry, E. E. et al. High conductivity in a nonplanar n-doped ambipolar semiconducting polymer. Chem. Mater. 29, 9742-9750 (2017).

19. Lin, X. et al. Beating the thermodynamic limit with photo-activation of n-doping in organic semiconductors. Nat. Mater. 16, 1209-1215 (2017).

20. Dixon, D. A., Calabrese, J. C. \& Miller, J. S. Crystal and molecular structure of the 2:1 charge-transfer salt of decamethylferrocene and perfluoro-7,7,8,8tetracyano-p-quinodimethane: $\left\{\left[\mathrm{Fe}\left(\mathrm{C}_{5} \mathrm{Me}_{5}\right)_{2}\right]^{\bullet-}\right\}_{2}\left[\mathrm{TCNQF}_{4}\right]^{2-}$. The electronic structure of $\left[\mathrm{TCNQF}_{4}\right]^{\mathrm{n}}(\mathrm{n}=0,1-, 2-)$. J. Phys. Chem. 93, 2284-2291 (1989).

21. Sutton, A. L. et al. Structural and optical investigations of charge transfer complexes involving the F4TCNQ dianion. CrystEngComm 16, 5234-5243 (2014).

22. Lu, J. et al. Synthetic precursors for $\mathrm{TCNQF}_{4}{ }^{2-}$ compounds: synthesis, characterization, and electrochemical studies of $\left(\mathrm{Pr}_{4} \mathrm{~N}\right)_{2} \mathrm{TCNQF}_{4}$ and $\mathrm{Li}_{2} \mathrm{TCNQF}_{4}$. J. Org. Chem. 77, 10568-10574 (2012).

23. Panja, S. et al. Dianions of 7,7,8,8-tetracyano-p-quinodimethane and perfluorinated tetracyanoquinodimethane: information on excited states from lifetime measurements in an electrostatic storage ring and optical absorption spectroscopy. J. Chem. Phys. 127, 124301 (2007).

24. Ma, L. et al. Single photon triggered dianion formation in TCNQ and F4TCNQ crystals. Sci. Rep. 6, 28510 (2016)

25. Cochran, J. E. et al. Molecular interactions and ordering in electrically doped polymers: blends of PBTTT and F4TCNQ. Macromolecules 47, 6836-6846 (2014)

26. Mendez, H. et al. Charge-transfer crystallites as molecular electrical dopants. Nat. Commun. 6, 8560 (2015).

27. Yim, K. H. et al. Controlling electrical properties of conjugated polymers via a solution-based p-type doping. Adv. Mater. 20, 3319-3324 (2008).

28. Karpov, Y. et al. High conductivity in molecularly p-doped diketopyrrolopyrrole-based polymer: the impact of a high dopant strength and good structural order. Adv. Mater. 28, 6003-6010 (2016).

29. Kroon, R. et al. Polar side chains enhance processability, electrical conductivity, and thermal stability of a molecularly p-doped polythiophene. Adv. Mater. 29, 1700930 (2017).

30. Liu, J. et al. Enhancing molecular n-type doping of donor-acceptor copolymers by tailoring side chains. Adv. Mater. 30, 1704630 (2018)

31. Li, J. et al. The effect of thermal annealing on dopant site choice in conjugated polymers. Org. Electron. 33, 23-31 (2016).

32. Song, C. K., Eckstein, B. J., Tam, T. L., Trahey, L. \& Marks, T. J. Conjugated polymer energy level shifts in lithium-ion battery electrolytes. ACS Appl. Mater. Interfaces 6, 19347-19354 (2014).
33. Moia, D. et al. A salt water battery with high stability and charging rates made from solution processed conjugated polymers with polar side chains. Preprint at http://arxiv.org/abs/1711.10457 (2017).

34. Brebels, J., Manca, J. V., Lutsen, L., Vanderzande, D. \& Maes, W. High dielectric constant conjugated materials for organic photovoltaics. J. Mater. Chem. A 5, 24037-24050 (2017).

35. Giovannitti, A. et al. N-type organic electrochemical transistors with stability in water. Nat. Commun. 7, 13066 (2016).

36. Giovannitti, A. et al. Controlling the mode of operation of organic transistors through side-chain engineering. Proc. Natl Acad. Sci. USA 113, 12017-12022 (2016)

37. Nielsen, C. B. et al. Molecular design of semiconducting polymers for high-performance organic electrochemical transistors. J. Am. Chem. Soc. 138, 10252-10259 (2016)

38. Glaudell, A. M., Cochran, J. E., Patel, S. N. \& Chabinyc, M. L. Impact of the doping method on conductivity and thermopower in semiconducting polythiophenes. Adv. Energy Mater. 5, 1401072 (2015).

39. Haworth, N. L. et al. Diagnosis of the redox levels of $\mathrm{TCNQF}_{4}$ compounds using vibrational spectroscopy. ChemPlusChem 79, 962-972 (2014).

40. Mityashin, A. et al. Unraveling the mechanism of molecular doping in organic semiconductors. Adv. Mater. 24, 1535-1539 (2012).

41. Patel, S. N. et al. Morphology controls the thermoelectric power factor of a doped semiconducting polymer. Sci. Adv. 3, e1700434 (2017)

42. Li, J. et al. Introducing solubility control for improved organic p-type dopants. Chem. Mater. 27, 5765-5774 (2015).

43. Bubnova, O. et al. Semi-metallic polymers. Nat. Mater. 13, 190-194 (2014).

44. Zuo, G., Abdalla, H. \& Kemerink, M. Impact of doping on the density of states and the mobility in organic semiconductors. Phys. Rev. B 93, 235203 (2016)

\section{Acknowledgements}

We gratefully acknowledge financial support from the Swedish Research Council through grant no. 2016-06146, the Knut and Alice Wallenberg Foundation through a Wallenberg Academy Fellowship and the European Research Council (ERC) under grant agreement no. 637624. The authors thank the Cornell High Energy Synchrotron Source (CHESS) (supported by the NSF \& NIH/NIGMS through NSF award DMR1332208) for providing experimental time for GIWAXS measurements. We thank the Freiburg Materials Research Center (FMF) and Anders Mårtensson (Chalmers) for help with SEC measurements. We would like to thank Professor Koen Vandewal for helpful discussions. S.R.M. and Y.Z. thank the US National Science Foundation for support of this work, under award no. DMR-1729737. S.F. and H.S. acknowledge financial support from VINNOVA (grant no. 2015-04859) and the Swedish Research Council (grant no. 2016-03979). DFT simulations by T.F.H., D.N. and A.J.M. were supported by the US Department of Energy, Office of Basic Energy Sciences under award DE-SC0010419. M.F. and X.L. acknowledge support by the Swedish Research Council (grant no. 201605498). A.G. and I.M. acknowledge funding from the Engineering and Physical Sciences Research Council (EP/G037515/1).

\section{Author contributions}

D.K., R.K. and C.M. conceived the project. R.K., A.G., D.S. and Y.Z. synthesized the materials. D.K., A.C. and J.H. prepared samples, performed electrical and spectroscopic measurements and analysed data. D.K. and L.Y. recorded and analysed the GIWAXS data. A.I.H., X.L. and M.F. recorded and analysed UPS spectra. H.S. conducted temperature-dependent conductivity and dielectric constant measurements. A.I.H. and A.G. performed the cyclic voltammetry measurements. T.F.H., D.N. and A.J.M. carried out DFT calculations and M.K. performed kinetic Monte Carlo modelling. D.K. A.I.H. and C.M. wrote the manuscript. S.R.M., I.M., M.F., S.F., M.S. and all the authors contributed to the data analysis, discussion and manuscript preparation.

\section{Competing interests}

The authors declare no competing interests.

\section{Additional information}

Supplementary information is available for this paper at https://doi.org/10.1038/ s41563-018-0263-6.

Reprints and permissions information is available at www.nature.com/reprints. Correspondence and requests for materials should be addressed to D.K. or C.M. Publisher's note: Springer Nature remains neutral with regard to jurisdictional claims in published maps and institutional affiliations.

(C) The Author(s), under exclusive licence to Springer Nature Limited 2019 


\section{Methods}

Materials. p(g $\mathrm{g}_{4}$ 2T-TT) was synthesized as described in the Supplementary Information, Section $1 ; \mathrm{p}(\mathrm{a} 2 \mathrm{~T}-\mathrm{TT})$ and $\mathrm{p}\left(\mathrm{g}_{3} 2 \mathrm{~T}-\mathrm{TT}\right)$ were synthesized according to a reported procedure ${ }^{36} \cdot \mathrm{pBTTT}(-\mathrm{C} 14$ ) (weight-average molecular weight $M_{\mathrm{w}} \sim 34 \mathrm{~kg} \mathrm{~mol}^{-1}$, dispersity $Ð \sim 2.2$ ) was purchased from Sigma Aldrich and used as received. F2TCNQ and F4TCNQ were purchased from TCI Chemicals and used as received without further purification. F6TCNNQ was synthesized according to a reported procedure ${ }^{45}$, and purified by sublimation at $2.5 \times 10^{-5}$ torr and $270^{\circ} \mathrm{C}$. The synthesis of $\mathrm{Li}^{+} \mathrm{F} 4 \mathrm{TCNQ}{ }^{--}$and $2 \mathrm{Li}^{+} \mathrm{F} 4 \mathrm{TCNQ}^{2-}$ is described in the Supplementary Information, Section 2. The solvents chloroform $\left(\mathrm{CHCl}_{3}\right.$; purity $>99 \%$ ), acetonitrile $\left(\mathrm{CH}_{3} \mathrm{CN} ; 99.9 \%\right.$, extra dry, over molecular sieves, AcroSeal $^{\varpi}$ ), chlorobenzene, 1,2-dichlorobenzene and dimethylformamide were purchased from Fisher Scientific.

Molecular weight determination. The molecular weight of $\mathrm{p}\left(\mathrm{g}_{4} 2 \mathrm{~T}-\mathrm{TT}\right)$ was found to be untypically high (outside the calibration range) when measured by size exclusion chromatography (SEC) in chloroform, indicating strong aggregation. Exchanging the solvent with $\mathrm{N}, \mathrm{N}$-dimethylacetamide with $0.5 \mathrm{wt} \% \mathrm{LiBr}$ at a temperature of $85^{\circ} \mathrm{C}$ yielded a number-average molecular weight $M_{\mathrm{n}} \sim 5 \mathrm{~kg} \mathrm{~mol}^{-1}$ for relative calibration against a poly(methyl methacrylate) standard (Supplementary Fig. 21). p(a2T-TT) was soluble in chloroform $\left(M_{\mathrm{n}}=3.5 \mathrm{~kg} \mathrm{~mol}^{-1}\right.$ and $M_{\mathrm{w}}=5.0 \mathrm{~kg} \mathrm{~mol}^{-1}$ ). SEC of pBTTT was performed on an Agilent PL-GPC 220 integrated high-temperature GPC/SEC system in 1,2,4-trichlorobenzene at $150^{\circ} \mathrm{C}$ using relative calibration with polystyrene standards.

Elemental analysis. Measurements of $\mathrm{p}\left(\mathrm{g}_{4} 2 \mathrm{~T}-\mathrm{TT}\right)$ were conducted with a sample size of $2 \mathrm{mg}$ on a vario MICRO cube elemental analyser (Elementar).

Sample fabrication. $\mathrm{p}\left(\mathrm{g}_{4} 2 \mathrm{~T}-\mathrm{TT}\right)$ and F4TCNQ were dissolved at room temperature to concentrations of $10 \mathrm{gl}^{-1}$ and $5 \mathrm{gl}^{-1}$ in a 1:1 mixture of $\mathrm{CH}_{3} \mathrm{CN}$ and $\mathrm{CHCl}_{3}$. $\mathrm{CH}_{3} \mathrm{CN}$ was chosen as a cosolvent because of its significantly higher solubility for the dopants used. Appropriate volumes of the $1: 1 \mathrm{CH}_{3} \mathrm{CN} / \mathrm{CHCl}_{3}$ mixture were added to the $\mathrm{p}\left(\mathrm{g}_{4} 2 \mathrm{~T}-\mathrm{TT}\right)$ solution before addition of the F4TCNQ, F2TCNQ or F6TCNNQ solution to assure similar polymer concentrations of $2-5 \mathrm{gl}^{-1}$ in the samples. $\mathrm{Li}^{+} \mathrm{F} 4 \mathrm{TCNQ}{ }^{-}$was dissolved at a concentration of $1 \mathrm{gl}^{-1}$ and appropriate amounts were added to $\mathrm{p}\left(\mathrm{g}_{4} 2 \mathrm{~T}-\mathrm{TT}\right)$ solutions. $\mathrm{p}(\mathrm{a} 2 \mathrm{~T}-\mathrm{TT})$ was dissolved in chloroform at a concentration of $5 \mathrm{gl}^{-1}$ and appropriate amounts of F4TCNQ or F6TCNNQ dissolved in $\mathrm{CHCl}_{3}$ at a concentration of $0.5 \mathrm{gl}^{-1}$ were added. pBTTT was dissolved in $1: 1$ chlorobenzene/o-dichlorobenzene at $80^{\circ} \mathrm{C}$ to a concentration of $5 \mathrm{gl}^{-1}$ and mixed with appropriate concentrations of F4TCNQ or F6TCNNQ dissolved at a concentration of $0.2 \mathrm{gl}^{-1}$ in the same solvent mixture. Thin films (thickness $50-250 \mathrm{~nm}$ ) were spin coated ( $1000 \mathrm{rpm}$ for $60 \mathrm{~s}$ ) on precleaned (acetone, isopropanol) microscopy glass slides for electrical measurements, n-doped silicon substrates for X-ray scattering or ITO glass for XPS measurements. Thicker samples $(1-3 \mu \mathrm{m})$ were prepared by drop casting onto cleaned PET substrates for Seebeck measurements or $\mathrm{CaF}_{2} / \mathrm{BaF}_{2}$ windows for FTIR solid absorption measurements, followed by solvent evaporation at ambient temperature and $80^{\circ} \mathrm{C}$ for $\mathrm{CH}_{3} \mathrm{CN} / \mathrm{CHCl}_{3}$ and chlorobenzene/o-dichlorobenzene, respectively. Sequentially doped samples were prepared by first spin coating thin films from polymer solutions $\left(5 \mathrm{gl}^{-1}\right)$ on $\mathrm{BaF}_{2}$ windows and subsequently doped by spreading $0.1 \mathrm{ml}$ of F4TCNQ solution in acetonitrile $\left(0.01 \mathrm{gl}^{-1}\right)$ on the polymer film, followed by spinning off the excess solvent.

UV-vis absorption spectroscopy. A PerkinElmer Lambda 900 spectrophotometer was used to measure absorption spectra of liquid and solid samples. We used leastsquare fitting of UV-vis spectra in the range between 2.5 and $4.0 \mathrm{eV}$ to quantify the different species of F4TCNQ. The spectra were fitted with the absorption coefficients of (1) F4TCNQ, (2) $\mathrm{Li}^{+} \mathrm{F} 4 \mathrm{TCNQ}{ }^{\bullet-}$, (3) $2 \mathrm{Li}^{+} \mathrm{F} 4 \mathrm{TCNQ}^{2-}$ and (4) a Gaussian centred at $2.4 \mathrm{eV}$, representing the contribution from amorphous $\mathrm{p}\left(\mathrm{g}_{4} 2 \mathrm{~T}-\mathrm{TT}\right)$.

FTIR spectroscopy. Infrared absorption spectra were recorded with a PerkinElmer Frontier FTIR spectrometer. The IR absorptions between 2,000 and $2,500 \mathrm{~cm}^{-1}$ of F4TCNQ dissolved in dry acetonitrile at a concentration of $5 \mathrm{gl}^{-1}$ as well as $\mathrm{Li}^{+} \mathrm{F} 4 \mathrm{TCNQ}{ }^{--}$and $2 \mathrm{Li}^{+} \mathrm{F} 4 \mathrm{TCNQ}^{2-}$, both a concentration of $0.3 \mathrm{gl}^{-1}$, were measured between two $\mathrm{BaF}_{2}$ or $\mathrm{CaF}_{2}$ windows with a $170 \mu \mathrm{m}$ thick polytetrafluoroethylene spacer. All FTIR spectra of doped polymers were corrected by removing the underlying polaron signal, which was interpolated by fitting a straight line to data points immediately outside the peak area (see Supplementary Fig. 32).

Electrical characterization. A four-point probe set-up from Jandel Engineering (cylindrical probe head, RM3000) with collinear tungsten carbide electrodes at a regular spacing of $1 \mathrm{~mm}$ and a fixed weight of $60 \mathrm{~g}$ was used to measure the resistance. The electrical conductivity was then calculated taking into account the thickness and a correction factor of 4.53. The thicknesses of drop-cast and spincoated films were determined with a microcaliper and a KLA Tencor AlphaStep D-100 profilometer, respectively. An SB1000 instrument (MMR Technologies) was used to measure the Seebeck coefficient at $300 \mathrm{~K}$ controlled with a K2000 temperature controller (MMR Technologies). For the measurements, samples of drop-cast films were cut $(1 \mathrm{~mm} \times 4 \mathrm{~mm})$ and connected to the sample stage with silver paint (Agar silver paint, G302), and a temperature difference of 1-2 K was applied. Constantan wire was used as an internal reference.

GIWAXS. Measurements using synchrotron radiation at a wavelength of $1.16 \AA$ were performed at the D-line of the Cornell High Energy Synchrotron Source (CHESS) at Cornell University. 2D scattering images were obtained with a Pilatus $200 \mathrm{~K}$ detector (pixel size of $172 \mu \mathrm{m} \times 172 \mu \mathrm{m}$ ) placed at a distance of $177.2 \mathrm{~mm}$ from the sample.

UPS. Measurements were carried out in a UHV surface analysis system $\left(1 \times 10^{-10} \mathrm{mbar}\right)$ equipped with a Scienta-200 hemispherical analyser, using a $\mathrm{He}-$ discharge lamp $(h \nu(\mathrm{He} \mathrm{I})=21.22 \mathrm{eV})$ as excitation source. The total experimental energy resolution was $80 \mathrm{meV}$ and all measurements were calibrated with respect to the Fermi level. To determine the energy resolution and to calibrate the work function, in situ sputter-cleaned gold surfaces were used. Samples were prepared in ambient conditions via spin coating of the respective polymer solution on ITO substrates (see section on sample fabrication). To limit exposure to oxygen, water and contaminants, the samples were stored under argon atmosphere until the measurement.

Cyclic voltammetry. Measurements of the dopants were performed on freshly prepared solutions of tetra- $n$-butylammonium hexafluorophosphate $\left(\mathrm{TBAPF}_{6}\right)$ in dry acetonitrile $(0.1 \mathrm{M})$ with analyte concentrations of $0.5 \mathrm{mM}$ to $1 \mathrm{mM}$ using a three-electrode set-up, consisting of Pt wires as counter- and working electrode and a $\mathrm{Ag}$ wire as pseudo-reference electrode. The voltammograms were recorded at a scan rate of $100 \mathrm{mV} \mathrm{s}^{-1}$ and are referenced with respect to the half-wave potential of ferrocene, which was added as internal standard. The electron affinities of

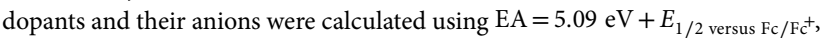
where $E_{1 / 2}$ versus $\mathrm{Fc} / \mathrm{Fc}^{+}$is the half-way potential versus ferrocene/ferrocene ${ }^{+}$ $\left(\mathrm{Fc} / \mathrm{Fc}^{+}\right)$. Thin films of $\mathrm{p}\left(\mathrm{g}_{4} 2 \mathrm{~T}-\mathrm{TT}\right), \mathrm{p}(\mathrm{a} 2 \mathrm{~T}-\mathrm{TT})$ and $\mathrm{pBTTT}$ were prepared by spin coating on ITO-coated glass substrates $\left(5 \mathrm{gl}^{-1}\right.$ in chloroform) and cyclic voltammetry measurements were carried out using a set-up as described above and a $0.1 \mathrm{M} \mathrm{TBAPF}_{6}$ acetonitrile solution as the supportive electrolyte with a scan rate of $100 \mathrm{mV} \mathrm{s}^{-1}$. The ionization energies of the polymers were calculated using $\mathrm{IE}=5.09 \mathrm{eV}+E_{\text {ox versus } \mathrm{Fc} / \mathrm{Fc}^{+}}$, where $E_{\text {ox versus } \mathrm{Fc} / \mathrm{Fc}^{+}}$is the oxidation onset versus $\mathrm{Fc} / \mathrm{Fc}^{+}$.

Impedance spectroscopy. Metal-insulator-semiconductor structures were fabricated to measure the low-frequency dielectric constant of the materials. pBTTT and $\mathrm{p}\left(\mathrm{g}_{4} 2 \mathrm{~T}-\mathrm{TT}\right)$ were dissolved in dichlorobenzene at a concentration of $5 \mathrm{gl}^{-1}$ and stirred overnight at $80^{\circ} \mathrm{C}$. The hot polymer solutions were spin coated onto precleaned $\mathrm{Si} / \mathrm{SiO}_{2}(200 \mathrm{~nm})$ substrates at $1000 \mathrm{rpm}$ and the resulting films were annealed at $120^{\circ} \mathrm{C}$ for $3 \mathrm{~h}$, with a thickness of $33 \pm 5 \mathrm{~nm}$ for pBTTT and $35 \pm 3 \mathrm{~nm}$ for $\mathrm{p}\left(\mathrm{g}_{4} 2 \mathrm{~T}-\mathrm{TT}\right)$ (measured by atomic force microscopy). Gold top electrodes $(40 \mathrm{~nm})$ with different surface areas were then evaporated through a shadow mask. Device fabrication was performed in a $\mathrm{N}_{2}$-filled glovebox. The impedance spectroscopy was performed in vacuum using an Alpha high-resolution dielectric analyser (Novocontrol), and for each area size six devices were measured. An a.c. voltage of $0.1 \mathrm{~V}$ and a d.c. voltage bias of $30 \mathrm{~V}$ were applied to ensure a fully depleted device. The depleted polymer layer acts as a capacitor in series with the oxide layer, and hence the total capacitance is

$$
\frac{1}{C_{\text {tot }}}=\frac{1}{C_{\mathrm{SiO}_{2}}}+\frac{1}{C_{\text {pol }}}
$$

where $C_{\mathrm{SiO}_{2}}$ and $C_{\mathrm{pol}}$ are the capacitances of $\mathrm{SiO}_{2}$ and the polymer. The latter is extracted by

$$
C_{\mathrm{pol}}=\frac{\varepsilon_{0} \varepsilon_{\mathrm{r}} A}{d}
$$

where $A$ is the device area, $\varepsilon_{\mathrm{r}}$ the polymer dielectric constant, $\varepsilon_{0}$ the vacuum permittivity and $d$ the polymer layer thickness.

For Mott-Schottky diode measurements thin films of $\mathrm{p}\left(\mathrm{g}_{4} 2 \mathrm{~T}-\mathrm{TT}\right)$ doped with $0.5 \mathrm{~mol} \%$ F4TCNQ $(70 \mathrm{~nm})$ and $\mathrm{Li}^{+} \mathrm{F} 4 \mathrm{TCNQ}{ }^{--}(55 \mathrm{~nm})$ were spin cast on p-doped $\mathrm{Si}$ wafers as bottom contacts (a thin layer of native $\mathrm{SiO}_{2}$ is expected on the surface) Devices with a working area of $9 \mathrm{~mm}^{2}$ were subsequently prepared by evaporation of aluminium top contacts $(70 \mathrm{~nm})$. Measurements were performed with a d.c. voltage varying from $-0.4 \mathrm{~V}$ to $0.4 \mathrm{~V}$, with $\mathrm{Si}$ acting as the ground electrode, and an a.c. voltage of $20 \mathrm{mV}$. For Mott-Schottky analysis we chose frequencies close to a phase angle of $-90^{\circ}\left(200 \mathrm{~Hz}\right.$ for $\mathrm{Li}^{+} \mathrm{F} 4 \mathrm{TCNQ}{ }^{--}$and $5 \mathrm{kHz}$ for F4TCNQ; Supplementary Fig. 28). The Mott-Schottky diodes were prepared in a $\mathrm{N}_{2}$-filled glovebox and the measurements were carried out in vacuum.

The free charge-carrier density, $N_{\mathrm{p}}$, was extracted as reported in ref. ${ }^{9}$.

$$
\frac{\mathrm{d}}{\mathrm{d} V} \frac{1}{C^{2}}=\frac{2}{q \varepsilon_{0} \varepsilon_{\mathrm{r}} A^{2}} \frac{1}{N_{\mathrm{p}}}
$$


where $C$ is the measured capacitance, $q$ the elementary charge and $A$ the device area.

Density functional theory (DFT) simulations. GaussView 6 was used to construct a dimer of $\mathrm{g}_{4} 2 \mathrm{~T}$-TT with full side chains on half of the dimer, and truncated side chains on the other half $\left(\mathrm{O}-\mathrm{CH}_{3}\right)$. An F4TCNQ molecule was intercalated between the full side chains, and DFT was performed using Gaussian 16 with the CAM-B3LYP functional and 6-31g(d) basis set and the Grimme D3 dispersion correction. The background dielectric constant was set to 7 using the polarizable continuum model. A 3-21g basis set was used to perform a rough geometry optimization before the more accurate $6-31 \mathrm{~g}(\mathrm{~d})$ basis was used for the final geometry optimization.

\section{Data availability}

The authors declare that the main data supporting the findings of this study are available within the article and its Supplementary Information files. Additional data are available from the corresponding authors upon request.

\section{References}

45. Koech, P. K. et al. Synthesis and application of 1,3,4,5,7,8-hexafluorotetracyanonaphthoquinodimethane (F6-TNAP): a conductivity dopant for organic light-emitting devices. Chem. Mater. 22, 3926-3932 (2010). 